\title{
Twisted reality condition for spectral triple on two points
}

\section{Ludwik Dąbrowski *}

SISSA (Scuola Internazionale Superiore di Studi Avanzati), Via Bonomea 265, 34136 Trieste, Italy

E-mail:dabrow@sissa.it

\section{Andrzej Sitarz}

Institute of Physics, Jagiellonian University, prof. Stanistawa Łojasiewicza 11, 30-348 Kraków, Poland. Institute of Mathematics of the Polish Academy of Sciences, Śniadeckich 8, 00-950 Warszawa, Poland. E-mail: andrzej.sitarz@uj.edu.pl

Lowest dimensional spectral triples with twisted reality condition over the function algebra on two points are discussed. The gauge perturbations (fluctuations), chiral gauge perturbations, conformal rescalings, and permutation of the two points are presented.

Proceedings of the Corfu Summer Institute 2015 "School and Workshops on Elementary Particle Physics and Gravity"

1-27 September 2015

Corfu, Greece

*L. Dąbrowski and A. Sitarz acknowledge support of the NCN grant 2012/06/M/ST1/00169 


\section{Introduction}

Real spectral triples should be understood as a noncommutative generalisation of spin manifolds, as proposed by Connes in [四] and demonstrated later in the reconstruction theorem [四]. In a recent paper [四, motivated by some examples of conformally rescaled noncommutative Dirac operators as well as examples arising from the quantum deformations we have proposed a new definition of reality condition, which includes a twist by an automorphism of the algebra ${ }^{1}$.

In this note we study the applications and consequences of the proposed definition for the finite spectral triple over the algebra of functions on two points, which is often taken as the simplest toy model for an almost noncommutative geometry and used to show the principles which are behind the noncommutative description of the Standard Model as arising from a discrete noncommutative geometry.

We will discuss systematically the lowest dimensional representations and the space of all Dirac operators with twisted and untwisted reality condition satisfied, due to the gauge perturbations (fluctuations), chiral gauge perturbations, conformal rescalings, and permutation of the two points. The formula for the distance between the two points will be presented.

\section{Twisted reality}

Let us recall the notion of real spectral triples with the twisted first order condition.

Definition 2.1. Let $v \in A u t(H)$ be a selfadjoint invertible operator on a Hilbert space $H$. Let $A$ be a complex $*$-algebra of operators on $H$, which is left invariant by the automorphism $v$, i.e. $v^{-1} a v \in A$ for any $a \in A$. We say that the spectral triple $(A, H, D)$ admits a $v$-twisted real structure if there exists an anti-linear map $J: H \rightarrow H$ such that $J^{*} J=\mathrm{id}, J^{2}=\varepsilon \mathrm{id}$, and, for all $a, b \in A$,

$$
\begin{gathered}
{\left[a, J b J^{-1}\right]=0,} \\
{[D, a] J v^{-2} b v^{2} J^{-1}=J b J^{-1}[D, a],} \\
D J v=\varepsilon^{\prime} v J D \\
v J v=J
\end{gathered}
$$

where $\varepsilon, \varepsilon^{\prime} \in\{+1,-1\}$.

If $(A, H, D)$ admits a grading operator $\gamma: H \rightarrow H, \gamma^{*}=\gamma, \gamma^{2}=\mathrm{id},[\gamma, a]=0$, for all $a \in A$, $\gamma D=-D \gamma$, and $v^{2} \gamma=\gamma v^{2}$, then the twisted real structure $J$ is also required to satisfy

$$
\gamma J=\varepsilon^{\prime \prime} J \gamma
$$

where $\varepsilon^{\prime \prime}$ is another sign.

This is a particular instance of Definition 2.1 in [四]. The signs $\varepsilon, \varepsilon^{\prime}, \varepsilon^{\prime \prime}$ determine the $K O$ dimension modulo 8 in the usual way [ [D] and the operator $J$ is antiunitary. In such a case we

\footnotetext{
${ }^{1}$ remaining in the framework of bona fide spectral triples in contrast to twisted spectral triples of the interesting recent paper [䧃]
} 
shall say that a spectral triple admits a $v$-twisted real structure, or simply that it is a $v$-twisted real spectral triple.

Often (R. (ل) is called the order-zero condition, (R.2) is called the twisted order-one condition, while we shall refer to ([2.3) as to the twisted $\varepsilon^{\prime}$-condition, and to ([2.4) as to the twisted regularity (with the adjective twisted omitted if $v=$ id).

Let $\Omega_{D}^{1}$ be a bimodule of one-forms:

$$
\Omega_{D}^{1}:=\left\{\sum_{i} \pi\left(a_{i}\right)\left[D, \pi\left(b_{i}\right)\right], a_{i}, b_{i} \in A\right\},
$$

where the sum is finite. By a fluctuated Dirac operator $D_{\alpha}$ we mean

$$
D_{\alpha}:=D+\alpha+\varepsilon^{\prime} v J \alpha J^{-1} v
$$

with the requirement that $\alpha$ is selfadjoint. We shall often use the shorthand notation $\alpha^{\prime}=v J \alpha J^{-1} v$. As shown in [四], $\left(A, H, D_{\alpha}\right)$ with (the same) $J$ is also a $v$-twisted real spectral triple. If $(A, H, D)$ is even with grading $\gamma$, then $\left(A, H, D_{\alpha}\right)$ is even with (the same) grading $\gamma$. Furthermore

$$
\Omega_{D_{\alpha}}^{1}=\Omega_{D}^{1}
$$

and the twisted fluctuations with composition form a semigroup. They correspond to "gauge transformations" in physics.

Note that it is also possible to modify the Dirac operator by a chiral gauge perturbation of the form:

$$
D_{A}^{\gamma}=D+\gamma A+\varepsilon^{\prime} J \gamma A J^{-1}
$$

where $A \in \Omega_{D}^{1}$ is antihermitian.

Another type of transformations are conformal rescalings by a positive element taken originally [3] from the algebra $A$, or [5] from $J A J^{-1}$. Here we take $k \in A$ to be not only positive but also invertible and such that $k^{-1}$ is also bounded. Denote $k_{J}:=J \mathrm{~kJ}^{-1}$. As shown in [W], given a real spectral triple $(A, H, D, J)$, for

$$
D_{k_{J}}=k_{J} D k_{J}, \quad v(h)=\left(k^{-1} k_{J}\right)(h),
$$

the datum $\left(A, H, J, D_{k_{J}}, v\right)$ is a $v$-twisted real spectral triple. If furthermore $(A, H, D, J)$ is even with grading $\gamma$, then $\left(A, H, D_{k_{J}}, J, v\right)$ is even with (the same) grading $\gamma$, and has the same KO-dimension as $(A, H, D, J)$. Furthermore, if $(A, H, D, J, v)$ is a $v$-twisted real spectral triple which satisfies the twisted order-one condition, then, for all $k$ as above such that $\bar{v}\left(k k_{J}\right)=k k_{J},\left(A, H, D_{k_{J}}, J, \mu\right)$ is a $\mu$-twisted real spectral triple, where

$$
D_{k_{J}}=k_{J} D k_{J}, \quad \mu(h)=k_{J} v k^{-1}(h) .
$$

The grading $\gamma$, if exists, is again unchanged.

We also note that

$$
\Omega_{D_{k_{J}}}^{1}=\Omega_{D}^{1},
$$


and conformal twist by $k$ of gauge fluctuated by $A$ (untwisted) real spectral triple is a gauge fluctuation by $B=k^{-1} A k^{-1}$ of conformally twisted by $k$ spectral triple. In particular, with $v$ as in (B.5)), we have for the corresponding Dirac operators

$$
D_{k_{J}}+A+\varepsilon^{\prime} v J A J^{-1} v=\left(D+B+\varepsilon^{\prime} J B J^{-1}\right)_{k_{J}},
$$

provided that $A=k B k$.

Before we proceed with the simplest examples of finite spectral triples let us recall and important notion of irreducibility of a (real) spectral triple.

Definition 2.2. A real spectral triple over an algebra $A$ is irreducible if the representation of algebra generated by $\gamma, a,[D, b], a, b \in A$ is not reducible.

Note that this definition obviously implies irreducibility of all the data (a weaker condition). In particular, it is easy to find examples of classical spectral triples over manifolds, which are irreducible with $J$ included but are reducible in the sense of the definition above. In this case $H$ would correspond to "charge $n$ plus $-n$ " fields while in our case to "charge zero" fields.

In this paper we shall study the simplest (nontrivial) possible example, which is the algebra of functions over two points. We shall work with its faithful representation on a Hilbert space, or what is the same with its isomorphic copy of operators. We will be interested whether it admits an irreducible real spectral triple and discuss the space of all Dirac operators with twisted and untwisted reality condition satisfied.

\section{A spectral triple over two points}

The $*$-algebra $A_{2}$ of complex valued functions over two points is isomorphic to $\mathbb{C}^{2}=\left\{\left(c_{+}, c_{-}\right)\right\}$ with $*$ acting as the complex conjugation. We will use the vector space basis given by the projection $e=e^{*}=e^{2}=(1,0)$ and $1-e$, where 1 is the unity (identity) element. An arbitrary element of the algebra can be written as

$$
a=c_{+} e+c_{-}(1-e) .
$$

Before we start with the construction of spectral triples let us recall the following fact:

Lemma 3.1. The only nondegenerate first order differential calculus over $A_{2}$ is the universal differential calculus, with:

$$
d\left(c_{1} e+c_{2}(1-e)\right)=c_{1} d e-c_{2} d e,
$$

with the bimodule of one-forms generated by de.

$$
e d e=d e(1-e) .
$$

The involution on the algebra extends to the one forms: $d e^{*}=-d e$.

As a corollary, for any given $D$, we have

$$
e[D, a]=[D, a](1-e), \quad \forall a \in A .
$$


Furthermore a general one-form $A \in \Omega_{D}^{1}$ can be parametrized by two complex numbers $\phi_{1}, \phi_{2}$ as follows

$$
A=\left(\phi_{1} e+\phi_{2}(1-e)\right)[D, e]
$$

The selfadjoint $A=A^{*}$ correspond to $\phi_{2}=-\phi_{1}^{*}$.

Note also that for $a=c_{+} e+c_{-}(1-e)$ we have:

$$
[D, a]=\left(c_{+}-c_{-}\right)[D, e] .
$$

Recalling the expression for the distance between the two points in terms of the spectral data:

$$
d_{D}:=\sup _{a \in A_{2}}\left\{\left|c_{+}-c_{-}\right| \mid\|[D, a]\| \leq 1\right\}
$$

from (B.4) we thus obtain that

$$
d_{D}=\frac{1}{\|[D, e]\|}
$$

We observe that the algebra $A_{2}$ has only one nontrivial automorphism corresponding to the permutation of the two points, $v(e)=1-e$, so that $v^{2}=\mathrm{id}$. Hence, the twisted order one condition (2.2) is identical to the usual order one condition. The same holds for the commutation relation of $v^{2}$ with $\gamma$. However the $\varepsilon^{\prime}$-condition $(\sqrt{2.3})$ and the twisted regularity (2.4) are different in the untwisted and twisted case, which influences the possible form of the other data.

\subsection{The minimal representation $\left(\mathbb{C}^{2}\right)$}

The lowest possible dimension of a faithful representation of the algebra $A_{2}$ is 2 . The canonical action (faithful representation) of $A_{2}$ on $\mathbb{C}^{2}$ is through diagonal matrices in $M_{2}(\mathbb{C})$. Therefore $A_{2}$ is necessarily its own commutant and although there are several inequivalent possibilities for the real structure $J$, for all of them we necessarily have that $J a J^{*}$ is an element of the algebra itself. Therefore, the order one condition ([2.2) in its twisted or untwisted version would contradict the bimodule relation between algebra elements and one-forms (B.3).

Hence, we reach the following conclusion:

Lemma 3.2. There are no irreducible real spectral triples (twisted or untwisted) with the Hilbert space $H=\mathbb{C}^{2}$ over the algebra $A_{2}=\mathbb{C}^{2}$, that give a non-zero differential calculus.

Of course, if we discard the real structure altogether we still have an irreducible spectral triple.

\section{The minimal even spectral triples $\left(\mathbb{C}^{3}\right)$}

The first possibility to have a nontrivial real (even) spectral triple with a faithful representation of $A_{2}$, is on $\mathbb{C}^{3}$ with the $\mathbb{Z}_{2}$-grading $\gamma$, which we take in the diagonal form

$$
\gamma=\left(\begin{array}{ccc}
1 & 0 & 0 \\
0 & -1 & 0 \\
0 & 0 & -1
\end{array}\right)
$$


and the representation of $A_{2}$ :

$$
A_{2} \ni a=\left(\begin{array}{ccc}
c_{+} & 0 & 0 \\
0 & c_{+} & 0 \\
0 & 0 & c_{-}
\end{array}\right) .
$$

The Dirac operator is a priori an arbitrary selfadjoint matrix in $M_{3}(\mathbb{C})$, however, using its anticommutation relation with $\gamma$ we are restricted to the following form:

$$
D=\left(\begin{array}{ccc}
0 & d_{2} & d_{1} \\
d_{2}^{*} & 0 & 0 \\
d_{1}^{*} & 0 & 0
\end{array}\right),
$$

where $d_{1}, d_{2} \in \mathbb{C}$.

\subsection{The distance formula}

Before we pass to real structures and the restrictions on $D$ imposed by ([2.2) and ([2.3]) we compute the one-forms $A \in \Omega_{D}^{1}$ associated to $D$. For that it suffices to compute

$$
[D, e]=\left(\begin{array}{ccc}
0 & 0 & -d_{1} \\
0 & 0 & 0 \\
d_{1}^{*} & 0 & 0
\end{array}\right),
$$

where

$$
e=\left(\begin{array}{lll}
1 & 0 & 0 \\
0 & 1 & 0 \\
0 & 0 & 0
\end{array}\right) .
$$

Then it follows that a general one-form $A$ can be parametrized by two complex numbers $\phi_{1}, \phi_{2}$ as follows

$$
A=\left(\begin{array}{ccc}
0 & 0 & -\phi_{1} d_{1} \\
0 & 0 & 0 \\
\phi_{2} d_{1}^{*} & 0 & 0
\end{array}\right)
$$

The selfadjoint $A=A^{*}$ correspond to $\phi_{2}=-\phi_{1}^{*}$.

Since the norm of $[D, a]$ is

$$
\|[D, a]\|=\left|c_{+}-c_{-}\right|\left|d_{1}\right|,
$$

it follows then that the distance (B.5) between the two points equals

$$
d_{D}=\frac{1}{\|[D, e]\|}=\frac{1}{\left|d_{1}\right|} .
$$

\subsection{Real structure}

It is easy to see that $[[D, e], e] \neq 0$ unless $d_{1}=d_{2}=0$. Thus to have the first order condition satisfied, $J$ cannot commute with $e$ and we cannot have $J A_{2} J \subset A_{2}$. The only (up to a simple rescaling) candidate for such $J$ is,

$$
J=\left(\begin{array}{lll}
1 & 0 & 0 \\
0 & 0 & 1 \\
0 & 1 & 0
\end{array}\right) \circ * .
$$


It satisfies $J^{2}=$ id and $J \gamma=\gamma J$. We note that $J a^{*} J$ becomes

$$
J a^{*} J=\left(\begin{array}{ccc}
c_{+} & 0 & 0 \\
0 & c_{-} & 0 \\
0 & 0 & c_{+}
\end{array}\right)
$$

Now, concerning the order one condition, it suffices to be checked only for $a=b=e$. Indeed a simple computation shows that it is satisfied:

$$
\left[[D, e], \mathrm{JeJ}^{-1}\right]=0 .
$$

independently of any other requirements.

However, to have a family o real (possibly twisted) spectral triples, we need to investigate possible twists.

\subsection{A family of real, conformally twisted, spectral triples.}

Let us begin by finding a family of Dirac operators compatible with $J$. We have:

$$
D J=\left(\begin{array}{ccc}
0 & d_{1} & d_{3} \\
d_{3}^{*} & 0 & 0 \\
d_{1}^{*} & 0 & 0
\end{array}\right) \circ *, \quad J D=\left(\begin{array}{ccc}
0 & d_{3}^{*} & d_{1}^{*} \\
d_{1} & 0 & 0 \\
d_{3} & 0 & 0
\end{array}\right) \circ *,
$$

so, imposing the commutation relation $J D=\varepsilon^{\prime} D J$ (the $\varepsilon^{\prime}$-condition), we obtain:

$$
d_{3}=\varepsilon^{\prime} d_{1}^{*}
$$

The self-adjoint one-forms

$$
A=\left(\phi e-\phi^{*}(1-e)\right)[D, e]=\left(\begin{array}{ccc}
0 & 0 & -\phi d_{1} \\
0 & 0 & 0 \\
-\phi^{*} d_{1}^{*} & 0 & 0
\end{array}\right)
$$

allow to fluctuate the Dirac operator by a real perturbation:

$$
A+\varepsilon^{\prime} J A J^{-1}=-\left(\begin{array}{ccc}
0 & \varepsilon^{\prime} \phi^{*} d_{1}^{*} & \phi d_{1} \\
\varepsilon^{\prime} \phi d_{1} & 0 & 0 \\
\phi^{*} d_{1}^{*} & 0 & 0
\end{array}\right)
$$

Hence starting from the Dirac operator $D$ with parameters $d_{1}$, by fluctuations (gauge perturbations) we obtain a family of gauge perturbed Dirac operators $D+A+\varepsilon^{\prime} J A J^{-1}$ parametrized by $(1-\phi) d_{1}$. It follows then from (3.5) and (4. (1) that

$$
d_{D+A+\varepsilon^{\prime} J A J^{-1}}=\frac{1}{|1-\phi|} d_{D}
$$

Concerning the perturbation of the type (2.6) we have the following observation

Remark 4.1. The family of chiral gauge perturbations $D+\gamma A+\varepsilon^{\prime} J \gamma A J^{-1}$ with $A=-A^{*} \in \Omega_{D}^{1}$, is equal to the family of usual gauge perturbations of $D$. $\diamond$ 
Now we shall consider a family of conformally rescaled Dirac operators. First of all, observe that there is no difference whether we use the rescaling by an element from the algebra or an element from the commutant.

Lemma 4.2. Let $k=\zeta(\rho e+(1-\rho)(1-e))$ and $h=\xi((1-\rho) e+\rho(1-e))$, where $\zeta, \xi>0$ and $0 \leq \rho \leq 1$. Let $k_{J}=J k^{-1}$. Then the conformal rescalings

$$
D_{k_{J}}=k_{J} D k_{J}, \quad D_{h}=h D h,
$$

of $D$ are identical provided that $\zeta^{2} \rho=(\xi)^{2}(1-\rho)$ and they correspond to the same twist:

$$
v=\left(\begin{array}{ccc}
1 & 0 & 0 \\
0 & \frac{1-\rho}{\rho} & 0 \\
0 & 0 & \frac{\rho}{1-\rho}
\end{array}\right)
$$

Proof. The proof is by explicit computation. First:

$$
k_{J}=\zeta\left(\begin{array}{ccc}
\rho & 0 & 0 \\
0 & (1-\rho) & 0 \\
0 & 0 & \rho
\end{array}\right), \quad h=\xi\left(\begin{array}{ccc}
(1-\rho) & 0 & 0 \\
0 & \rho & 0 \\
0 & 0 & (1-\rho)
\end{array}\right)
$$

out of which it is easy to see that indeed:

$$
\begin{gathered}
D_{k_{J}}=\zeta^{2}\left(\begin{array}{ccc}
0 & \rho(1-\rho) \varepsilon^{\prime} d_{1}^{*} & \rho^{2} d_{1} \\
\rho(1-\rho) \varepsilon^{\prime} d_{1} & 0 & 0 \\
\rho^{2} d_{1}^{*} & 0 & 0
\end{array}\right), \\
D_{h}=(\xi)^{2}\left(\begin{array}{ccc}
0 & (1-\rho)^{2} \varepsilon^{\prime} d_{1}^{*} \rho(1-\rho) d_{1} \\
(1-\rho)^{2} \varepsilon^{\prime} d_{1} & 0 & 0 \\
\rho(1-\rho) d_{1}^{*} & 0 & 0
\end{array}\right) .
\end{gathered}
$$

The formula for the twists follows directly.

As a result we can consider just one type of twists and using a general selfadjoint one-form (4.3) perform now fluctuations (gauge perturbations) of $D_{k_{J}}$ by

$$
A+\varepsilon^{\prime} v J A J^{-1} v=-\zeta^{2}\left(\begin{array}{ccc}
0 & \varepsilon^{\prime} \rho(1-\rho) \phi^{*} d_{1}^{*} & \rho^{2} \phi d_{1} \\
\varepsilon^{\prime} \rho(1-\rho) \phi d_{1} & 0 & 0 \\
\rho^{2} \phi^{*} d_{1}^{*} & 0 & 0
\end{array}\right)
$$

which are again parametrized by $\phi \in \mathbb{C}$.

Since the overall factor $\zeta$ can be incorporated into a redefined parameter $\phi$, by the composition of fluctuations (gauge perturbations) with conformal rescalings of (untwisted) real nondegenerate (that is $d_{1} \neq 0$ ) spectral triple, we obtain generically a 3-dimensional (real) parameter family of Dirac operators and thus of (twisted) spectral triples.

We remark that similarly to the untwisted case the chiral gauge perturbations are identical with the usual ones. 
Note also that the distance formula is only rescaled by $\rho \zeta$ :

$$
d_{D_{k_{J}}}=\frac{1}{\left\|\left[D_{k_{J}}, e\right]\right\|}=\frac{1}{\rho^{2} \zeta^{2}\left|d_{1}\right|},
$$

however, the place where the twist appears is in the respective powers of the Dirac operator:

$$
\left(D_{k_{J}}\right)^{2}=\zeta^{4} d_{1} d_{1}^{*}\left(\begin{array}{ccc}
\rho^{2}\left((1-\rho)^{2}+\rho^{2}\right) & 0 & 0 \\
0 & \rho^{2}(1-\rho)^{2} & 0 \\
0 & 0 & \rho^{4}
\end{array}\right) .
$$

\subsection{The permutation twist}

In Definition ] $\left[\right.$ we adopted the usual assumption about the twist $v$ that $v^{-1} \pi(a) v$ is $\pi(\bar{v}(a))$, where $\bar{v}$ is an automorphism of the algebra. In our the case with the chosen representation there are no such nontrivial maps $v$, however we can depart slightly from this assumption if the automorphism $v$ satisfies $v^{2}=1$. In that case the relation (2.2) is automatically satisfied. If we take

$$
v=\left(\begin{array}{lll}
1 & 0 & 0 \\
0 & 0 & 1 \\
0 & 1 & 0
\end{array}\right)
$$

then also the twisted regularity condition $v J v=J$ is satisfied.

Furthermore, we have:

$$
D J v=\left(\begin{array}{ccc}
0 & d_{2} & d_{1} \\
d_{2}^{*} & 0 & 0 \\
d_{1}^{*} & 0 & 0
\end{array}\right) \circ *, \quad v J D=\left(\begin{array}{ccc}
0 & d_{2}^{*} & d_{1}^{*} \\
d_{2} & 0 & 0 \\
d_{1} & 0 & 0
\end{array}\right) \circ *,
$$

so, imposing the twisted $\varepsilon^{\prime}$-condition, $D J v=\varepsilon^{\prime} v J D$, we obtain:

$$
d_{2}^{*}=\varepsilon^{\prime} d_{2}, \quad d_{1}^{*}=\varepsilon^{\prime} d_{1} .
$$

Hence the family of $v$-real Dirac operators is parametrized by $\left(d_{1}, d_{2}\right) \in \mathbb{R}$ (for $\varepsilon^{\prime}=1$ ) or $\left(d_{1}, d_{2}\right) \in$ $i \mathbb{R}\left(\right.$ for $\left.\varepsilon^{\prime}=-1\right)$ :

$$
\left(\begin{array}{ccc}
0 & d_{2} & d_{1} \\
\varepsilon^{\prime} d_{2} & 0 & 0 \\
\varepsilon^{\prime} d_{1} & 0 & 0
\end{array}\right)
$$

One can similarly as before compute the family of gauge fluctuated Dirac operators, which is parametrized by a complex number $\phi$ and amounts to the change:

$$
\left(d_{1}, d_{2}\right) \rightarrow\left(\left(1-\phi-\phi^{*}\right) d_{1}, d_{2}\right)
$$

The chiral gauge fluctuations are again exactly the same, so we see that only one parameter of the Dirac operator is changed via all possible gauge transformations.

The formula for the distance for the fluctuated $D$ is,

$$
d_{D+A+\varepsilon^{\prime} v J A J^{-1} v}=\frac{1}{\max \left\{\left|1-\phi-\phi^{*}\right|\left|d_{1}\right|,\left|d_{2}\right|\right\}} .
$$




\section{The spectral triples on $\mathbb{C}^{4}$}

The next possibility of a low-dimensional spectral triple, which is irreducible is with of $A_{2}$ on $\mathbb{C}^{4}$ and with the $\mathbb{Z}_{2}$-grading $\gamma$ such that $A_{2}$ has an irreducible representation on each eigenspace of $\gamma$ :

$$
\gamma=\left(\begin{array}{cccc}
1 & 0 & 0 & 0 \\
0 & -1 & 0 & 0 \\
0 & 0 & -1 & 0 \\
0 & 0 & 0 & 1
\end{array}\right), \quad A_{2} \ni a=\left(\begin{array}{cccc}
c_{+} & 0 & 0 & 0 \\
0 & c_{+} & 0 & 0 \\
0 & 0 & c_{-} & 0 \\
0 & 0 & 0 & c_{-}
\end{array}\right)
$$

The Dirac operator is a priori an arbitrary selfadjoint matrix in $M_{4}(\mathbb{C})$, however, using its anticommutation relation with $\gamma$ we are restricted to the following form:

$$
D=\left(\begin{array}{cccc}
0 & d_{3} & d_{1} & 0 \\
d_{3}^{*} & 0 & 0 & d_{2} \\
d_{1}^{*} & 0 & 0 & d_{4} \\
0 & d_{2}^{*} & d_{4}^{*} & 0
\end{array}\right)
$$

where $d_{1}, d_{2}, d_{3}, d_{4} \in \mathbb{C}$.

\subsection{The distance formula}

Before we pass to real structures and the restrictions on $D$ imposed by ([2.2) and (2.3]) we compute the one-forms $A \in \Omega_{D}^{1}$ associated to $D$. For that it suffices to compute

$$
[D, e]=\left(\begin{array}{cccc}
0 & 0 & -d_{1} & 0 \\
0 & 0 & 0 & -d_{2} \\
d_{1}^{*} & 0 & 0 & 0 \\
0 & d_{2}^{*} & 0 & 0
\end{array}\right)
$$

where

$$
e=\left(\begin{array}{llll}
1 & 0 & 0 & 0 \\
0 & 1 & 0 & 0 \\
0 & 0 & 0 & 0 \\
0 & 0 & 0 & 0
\end{array}\right)
$$

together with 1 , forms the basis of $A_{2}$. Then it follows that a general one-form $A$ can be parametrized by two complex numbers $\phi_{1}, \phi_{2}$ as follows

$$
A=\phi_{1} e[D, e]+\phi_{2}(1-e)[D, e]=\left(\begin{array}{cccc}
0 & 0 & -\phi_{1} d_{1} & 0 \\
0 & 0 & 0 & -\phi_{1} d_{2} \\
\phi_{2} d_{1}^{*} & 0 & 0 & 0 \\
0 & \phi_{2} d_{2}^{*} & 0 & 0
\end{array}\right)
$$

The selfadjoint $A=A^{*}$ correspond to $\phi_{1}=-\phi_{2}^{*}=: \phi$.

We also note that for $a=c_{+} e+c_{-}(1-e)$ :

$$
[D, a]=\left(c_{+}-c_{-}\right)[D, e] .
$$


The norm of $[D, a]$ is

$$
\|[D, a]\|=\left|c_{+}-c_{-}\right| \max \left\{\left|d_{1}\right|,\left|d_{2}\right|\right\},
$$

It follows then that the distance (B.5) between the two points equals

$$
d_{D}=\frac{1}{\|[D, e]\|}=\frac{1}{\max \left\{\left|d_{1}\right|,\left|d_{2}\right|\right\}} .
$$

\subsection{The real structure on $\mathbb{C}^{4}$.}

Again, as in the case previous case we look for possible $J$, antilinear isometries that map the algebra into the genuine commutant. Then, up to a unitary transform such $J$ takes the form

$$
J=\left(\begin{array}{llll}
1 & 0 & 0 & 0 \\
0 & 0 & 1 & 0 \\
0 & 1 & 0 & 0 \\
0 & 0 & 0 & 1
\end{array}\right) \circ *
$$

It satisfies $J^{2}=$ id and $J \gamma=\gamma J$. We note that $J a^{*} J$ becomes

$$
J a^{*} J=\left(\begin{array}{cccc}
c_{+} & 0 & 0 & 0 \\
0 & c_{-} & 0 & 0 \\
0 & 0 & c_{+} & 0 \\
0 & 0 & 0 & c_{-}
\end{array}\right)
$$

Now, concerning the order one condition, it suffices to be checked only for $a=b=e$. Indeed a simple computation shows that it is satisfied:

$$
\left[[D, e], \mathrm{JeJ}^{-1}\right]=0 .
$$

Next we shall impose the (possibly twisted) commutation relations (‥3]) and (․․ separately for the untwisted and twisted cases.

\subsection{Real spectral triples}

We begin with the untwisted case, first

$$
D J=\left(\begin{array}{cccc}
0 & d_{1} & d_{3} & 0 \\
d_{3}^{*} & 0 & 0 & d_{2} \\
d_{1}^{*} & 0 & 0 & d_{4} \\
0 & d_{4}^{*} & d_{2}^{*} & 0
\end{array}\right) \circ *, \quad J D=\left(\begin{array}{cccc}
0 & d_{3}^{*} & d_{1}^{*} & 0 \\
d_{1} & 0 & 0 & d_{4}^{*} \\
d_{3} & 0 & 0 & d_{2}^{*} \\
0 & d_{2} & d_{4} & 0
\end{array}\right) \circ *
$$

so, imposing the commutation relation $J D=\varepsilon^{\prime} D J$ (the $\varepsilon^{\prime}$-condition), we obtain:

$$
d_{3}=\varepsilon^{\prime} d_{1}^{*}, \quad d_{4}=\varepsilon^{\prime} d_{2}^{*}
$$

Having now fixed $d_{1}$ and $d_{2}$ we can consider the space of all real perturbations of the Dirac operator by one forms. The associated space of one forms $A$ can be parametrized by two complex numbers $\phi_{1}, \phi_{2}$, however a selfadjoint one-form has $\phi_{1}=-\phi_{1}^{*}=: \phi$, and we have 


$$
A=\left(\phi e-\phi^{*}(1-e)\right)[D, e]=-\left(\begin{array}{cccc}
0 & 0 & \phi d_{1} & 0 \\
0 & 0 & 0 & \phi d_{2} \\
\phi^{*} d_{1}^{*} & 0 & 0 & 0 \\
0 & \phi^{*} d_{2}^{*} & 0 & 0
\end{array}\right)
$$

and the real perturbation:

$$
A+\varepsilon^{\prime} J A J^{-1}=-\left(\begin{array}{cccc}
0 & \varepsilon^{\prime} \phi^{*} d_{1}^{*} & \phi d_{1} & 0 \\
\varepsilon^{\prime} \phi d_{1} & 0 & 0 & \phi d_{2} \\
\phi^{*} d_{1}^{*} & 0 & 0 & \varepsilon^{\prime} \phi^{*} d_{2}^{*} \\
0 & \phi^{*} d_{2}^{*} & \varepsilon^{\prime} \phi d_{2} & 0
\end{array}\right)
$$

Hence starting from the Dirac operator $D$ with parameters $\left(d_{1}, d_{2}\right)$, by fluctuations (gauge perturbations) we obtain a family of gauge perturbed Dirac operators $D+A+\varepsilon^{\prime} J A J^{-1}$ parametrized by $\left((1-\phi) d_{1},(1-\phi) d_{2}\right)$. It follows then from (5.1I) and (5.3) that

$$
d_{D+A+\varepsilon^{\prime} J A J^{-1}}=\frac{1}{|1-\phi|} d_{D} .
$$

The chiral gauge perturbations (2.6) with $A=-A^{*} \in \Omega_{D}^{1}$, become

$$
\gamma A+\varepsilon^{\prime} J \gamma A J^{-1}=-\left(\begin{array}{cccc}
0 & \varepsilon^{\prime} \phi^{*} d_{1}^{*} & \phi d_{1} & 0 \\
\varepsilon^{\prime} \phi d_{1} & 0 & 0 & -\phi d_{2} \\
\phi^{*} d_{1}^{*} & 0 & 0 & -\varepsilon^{\prime} \phi^{*} d_{2}^{*} \\
0 & -\phi^{*} d_{2}^{*} & -\varepsilon^{\prime} \phi d_{2} & 0
\end{array}\right),
$$

and we see that starting from the Dirac operator $D$ with parameters $\left(d_{1}, d_{2}\right)$, the chiral fluctuations (gauge perturbations) lead to a family parametrized by $\left((1-\phi) d_{1},-(1-\phi) d_{2}\right)$.

We can sum up the results of this subsection as

Lemma 5.1. In the real spectral triple over two points represented on $\mathbb{C}^{4}$ the entire family of Dirac operators $(D \neq 0)$ is obtained through gauge fluctuations and chiral gauge fluctuations starting from a nondegenerate spectral triple $\left(d_{1} \neq 0\right)$.

\subsection{The family of twisted real spectral triples.}

Since the reality operator is in fact very similar to the one in the $\mathbb{C}^{3}$ case, we find a similar property concerning conformal scaling by an element from the algebra and the one from the commutant.

Lemma 5.2. Let $k=\zeta(\rho e+(1-\rho)(1-e))$ and $h=\xi((1-\rho) e+\rho(1-e))$, where $\zeta, \xi>0$ and $0 \leq \rho \leq 1$. Let $k_{J}=J k^{-1}$. Then the conformal rescalings

$$
D_{k_{J}}=k_{J} D k_{J}, \quad D_{h}=h D h,
$$

of $D$ are identical provided that $\zeta^{2} \rho=(\xi)^{2}(1-\rho)$ and they correspond to the same twist:

$$
v=\left(\begin{array}{cccc}
1 & 0 & 0 & 0 \\
\rho 0 & \frac{1-\rho}{\rho} & 1 & 0 \\
0 & 0 & \frac{\rho}{1-\rho} & 0 \\
0 & 0 & 0 & 1
\end{array}\right)
$$


We skip the computational proof, and concentrate on the operator $D_{k_{J}}$,

$$
D_{k_{J}}=\zeta^{2}\left(\begin{array}{cccc}
0 & \rho(1-\rho) \varepsilon^{\prime} d_{1}^{*} & \rho^{2} d_{1} & 0 \\
\rho(1-\rho) \varepsilon^{\prime} d_{1} & 0 & 0 & (1-\rho)^{2} d_{2} \\
\rho^{2} d_{1}^{*} & 0 & 0 & \rho(1-\rho) d_{2}^{*} \varepsilon^{\prime} \\
0 & (1-\rho)^{2} d_{2}^{*} & \rho(1-\rho) \varepsilon^{\prime} d_{2} & 0
\end{array}\right)
$$

We can perform now fluctuations (gauge perturbations) of $D_{k_{J}}$ by the real gauge fields oneforms, which are again parametrized by $\phi \in \mathbb{C}$ :

$$
A+\varepsilon^{\prime} v J A J^{-1} v=-\zeta^{2}\left(\begin{array}{cccc}
0 & \varepsilon^{\prime} \rho(1-\rho) \phi^{*} d_{1}^{*} & \rho^{2} \phi d_{1} & 0 \\
\varepsilon^{\prime} \rho(1-\rho) \phi d_{1} & 0 & 0 & (1-\rho)^{2} \phi d_{2} \\
\rho^{2} \phi^{*} d_{1}^{*} & 0 & 0 & \varepsilon^{\prime} \rho(1-\rho) \phi^{*} d_{2}^{*} \\
0 & (1-\rho)^{2} \phi^{*} d_{2}^{*} & \varepsilon^{\prime} \rho(1-\rho) \phi d_{2} & 0
\end{array}\right)
$$

We close this section by giving the norm of $\left[D_{k_{J}}+A+\varepsilon^{\prime} v J A J^{-1} v, a\right]$

$$
\left.\left\|\left[D_{k_{J}}+A+\varepsilon^{\prime} v J A J^{-1} v, a\right]\right\|=\left|c_{+}-c_{-}\right| \zeta^{2}|1-\phi| \max \left\{\rho^{2}\left|d_{1}\right|,(1-\rho)^{2}\left|d_{2}\right|\right\}\right\},
$$

It follows then that the distance between the two points is:

$$
d_{D_{k_{J}}+A+\varepsilon^{\prime} v J A J^{-1} v}=\frac{1}{\|[D, e]\|}=\frac{1}{\zeta^{2}|1-\phi| \max \left\{\rho^{2}\left|d_{1}\right|,(1-\rho)^{2}\left|d_{2}\right|\right\}} .
$$

\subsection{The permutation twist}

We shall employ here the twist automorphism

$$
v=\left(\begin{array}{llll}
0 & 0 & 0 & 1 \\
0 & 0 & 1 & 0 \\
0 & 1 & 0 & 0 \\
1 & 0 & 0 & 0
\end{array}\right)
$$

which is involutive $v^{2}=\mathrm{id}$ and implements the automorphism that permutes (exchanges) $c_{-}$and $c_{+}$in $a \in A$.

A simple calculation shows that the twisted regularity condition

$$
v J v=J
$$

is satisfied.

We remark that the twist automorphism

$$
v^{\prime}=\left(\begin{array}{llll}
0 & 0 & 1 & 0 \\
0 & 0 & 0 & 1 \\
1 & 0 & 0 & 0 \\
0 & 1 & 0 & 0
\end{array}\right)
$$

that is also involutive $v^{2}=\mathrm{id}$ and implements the automorphism that permutes (exchanges) $c_{-}$and $c_{+}$in $a \in A$, is not suitable as it does not satisfy the twisted regularity condition. 
Furthermore, we have:

$$
D J v=\left(\begin{array}{cccc}
0 & d_{3} & d_{1} & 0 \\
d_{2} & 0 & 0 & d_{3}^{*} \\
d_{4} & 0 & 0 & d_{1}^{*} \\
0 & d_{2}^{*} & d_{4}^{*} & 0
\end{array}\right) \circ *, \quad v J D=\left(\begin{array}{cccc}
0 & d_{2} & d_{4} & 0 \\
d_{3} & 0 & 0 & d_{2}^{*} \\
d_{1} & 0 & 0 & d_{4}^{*} \\
0 & d_{3}^{*} & d_{1}^{*} & 0
\end{array}\right) \circ *,
$$

so, imposing the twisted $\varepsilon^{\prime}$-condition, $D J v=\varepsilon^{\prime} v J D$, we obtain:

$$
d_{3}=\varepsilon^{\prime} d_{2}, \quad d_{4}=\varepsilon^{\prime} d_{1}
$$

Hence the family of $v$-real Dirac operators is:

$$
\left(\begin{array}{cccc}
0 & \varepsilon^{\prime} d_{2} & d_{1} & 0 \\
\varepsilon^{\prime} d_{2}^{*} & 0 & 0 & d_{2} \\
d_{1}^{*} & 0 & 0 & \varepsilon^{\prime} d_{1} \\
0 & d_{2}^{*} & \varepsilon^{\prime} d_{1}^{*} & 0
\end{array}\right)
$$

Similarly as in the untwisted case the family of gauge fluctuated Dirac operators is parametrized by a complex number $\phi$ and amounts to the change:

$$
\left(d_{1}, d_{2}\right) \rightarrow\left((1-\phi) d_{1},(1-\phi) d_{2}\right)
$$

The formula for the distance is

$$
d_{D+A+\varepsilon^{\prime} v J A J^{-1} v}=\frac{1}{|1-\phi| \max \left\{\left|d_{1}\right|,\left|d_{2}\right|\right\}} .
$$

\subsection{Composition of twists}

Finally, since in the case of the $A_{2}$ algebra we have two different types of twists, the conformal twists and the permutation twist (in the $\mathbb{C}^{4}$ representation case) there is a natural question, whether these twists can be composed. However, explicit computations show:

Lemma 5.3. The composition of the conformal twists with the permutation twists does not satisfy the twisted regularity condition (2.4).

\section{Final remarks}

In this note a preparatory material has been presented for the study of more complicated finite dimensional examples, admitting non trivial twist automorphisms. Our interest is mainly in the finite spectral triple of the noncommutative version of the standard model, where the first order condition requires a particular attention. For that the product of (twisted) real spectral triples will need to be introduced and studied. It will be also interesting to study if the twist can be helpful with the reality condition satisfied only "up to infinitesimals" for some spectral triples on quantum groups.

In this note, we have worked out for the simplest nontrivial algebra and lowest dimensional representations and spectral triples of the two-point space, which often serves as a toy model for 
the finite part of Standard Model algebra (two-sheeted space). We have discussed the issue of the (usual and twisted) reality, conditions related to the conformal rescaling and to the permutation automorphism. The gauge perturbations (or "fluctuations") both usual and chiral have been explicitly computed and the dependence of the families of possible (twisted) Dirac operators have been recast in terms of the fluctuation parameters.

We have demonstrated that even in the simplest possible example of a spectral triple, the twists and the twisted reality conditions does indeed appear and need to be taken into account. These preliminary results aim to demonstrated the richness of the the theory as well as serve as the basis for future studies motivated by the noncommutative description of the elementary particle models.

\section{Acknowledgements}

L. Dabrowski and A. Sitarz acknowledge support of the NCN grant 2012/06/M/ST1/00169.

\section{References}

[1] T. Brzeziński, N. Ciccoli, L. Dabrowski, A. Sitarz, Twisted reality condition for Dirac operators, arXiv: 1410.6587

[2] A. Connes, Noncommutative geometry and reality, J. Math. Phys. 36, 6194-6231, (1995)

[3] A. Connes, P. Tretkoff, The Gauss-Bonnet theorem for the noncommutative two torus, In: Noncommutative Geometry, Arithmetic, and Related Topics, pp. 141-158. Johns Hopkins University Press (2011)

[4] A. Connes, On the spectral characterization of manifolds, J. Noncom. Geom. 7, 1-82 (2013)

[5] L. Dabrowski, A. Sitarz, Asymmetric noncommutative torus, SIGMA 11, 075-086, (2015)

[6] G. Landi, P. Martinetti, On twisting real spectral triples by algebra automorphisms, arXiv:1601.00219, 